\title{
Continuous Wave Pumped Supercontinuum Assisted by a Weak Femtosecond Pulse Seed
}

\author{
Peng Lu ${ }^{1}$, Qian Li ${ }^{1 *}$, Chao Huang ${ }^{1}$, K. Senthilnathan ${ }^{2}$ and K. Nakkeeran ${ }^{3}$ \\ ${ }^{1}$ School of Electronic and Computer Engineering, Peking University, Shenzhen, China \\ ${ }^{2}$ Department of Physics, School of Advanced Sciences, VIT University, Vellore 632 014, Tamil Nadu, India \\ ${ }^{3}$ School of Engineering, Fraser Noble Building, University of Aberdeen, Aberdeen AB24 3UE, U.K
}

E-mail: liqian@ece.pku.edu.cn

\begin{abstract}
We numerically investigate the effects of a weak coherent femtosecond pulse seed on the continuous wave $(\mathrm{CW})$ pumped supercontinuum (SC) generation. We analyze the evolution of CW pumped SC generation with various random noises and we observe the instabilities, which are initiated by modulation instability (MI), in the spectrum of the SC. To actively control the instabilities, we introduce a weak coherent femtosecond pulse seed to improve the properties of CW pumped SC in terms of spectral bandwidth and pulse-to-pulse stability. The detailed numerical results corroborate that the frequency shift and the peak power of the seed significantly decide the properties of the SC. The present investigation would highly be useful for realizing a broader CW pumped SC with enhanced coherence and SNR.
\end{abstract}

Keywords: supercontinuum, fibers, nonlinear optics, modulation instability

\section{Introduction}

Supercontinuum generation in optical fibers has gained a wide range of research interests and practical applications in many fields such as optical coherence tomography [1], optical frequency metrology [2], and generation of ultrashort optical pulses [3]. In general, the femtosecond (fs) pulses with peak power at kilo-watt level are used to pump SC generation [4 - 6]. However, the fs pulse pump is sensitive to perturbations and it requires exceptional dispersion control. Then, the picosecond even nanosecond pulses are utilized to pump SC generation $[6,7]$. At present, the picosecond pulse pumped SC is commercially available. Continuous wave (CW) has also been experimentally demonstrated for SC generation in the early 21 st century [8 - 12]. Compared to pulses pumped SC generation, CW pumped SC generation possesses some salient features. Generally, the setup of $\mathrm{CW}$ pumped SC consists of CW fiber laser that is spliced directly to a suitable optical fiber and hence it does not require any mechanically tunable components. In $\mathrm{CW}$ pumped SC generation, the stochastic nature of the modulation instability (MI) and the very large number of interacting solitons participating in the SC generation process lead to smooth continuum spectra. The CW lasers such as cascaded Raman fiber laser are capable of generating the multi-watts average output power while few pulses pumped SC are capable of achieving that $[5,12]$. Further, the average spectral power density of pulses pumped SC is in the range of -20 to -10 $\mathrm{dBm} / \mathrm{nm}[6-7,13-14]$ whereas the spectral power density of $\mathrm{CW}$ pumped $\mathrm{SC}$ exceeds $8 \mathrm{dBm} / \mathrm{nm}$ [7]. In addition, $\mathrm{CW}$ pumped SC also exhibits less amount of relative intensity noise of 30-50 dB and this range is lower than that of pump pulsed one [15]. Thus, the CW pumped SC has successfully been utilized to build the optical coherence tomography imaging [16].

The dynamics of CW pumped SC generation in optical fibers has been investigated both experimentally [7, 17 - 20] and numerically [21 - 25]. Unlike the fs pulse pumped SC, the $\mathrm{CW}$ pumped SC is initiated by MI process where noise plays an indispensable role. Here, MI helps in breaking CW into fundamental solitons. Further, the Raman self-frequency shift and Raman mediated soliton collisions lead to redshifting of the solitons. If the MI-induced solitons are close enough to the zero-dispersion wavelength (ZDW), blueshifting takes place due to dispersive waves. Owing to MI 
driven noise, the $\mathrm{CW}$ pumped $\mathrm{SC}$ exhibits significant pulseto-pulse fluctuations that results in the low mutual coherence, poor signal-to-noise ratio (SNR) and instability of peak power probability distribution. Mussot et al. have experimentally and numerically demonstrated that $\mathrm{CW}$ pumped SC is temporally characterized by strong and brief power fluctuations i.e. so-called optical rogue waves (ORW) that arise from soliton collisions [26]. It has been reported that the instability produced by MI can be controlled either by changing the input wavelength [27], pump pulse length [28], or adding a feedback [29]. Solli et al. have demonstrated that using a weak fs pulse seed on picosecond pulse pumped SC could produce an optically switchable, ultra-stable and bright SC with highly enhanced coherence [30]. Thus, the fs pulse seed will not only broaden the spectral bandwidth but also enhance the temporal coherence [28] and SNR [31].

In this paper, by launching a weak coherent fs pulse seed, we numerically investigate the effects of the fs seed on the properties of CW pumped SC. To be precise, we study the nature of fs seeded SC by varying the frequency shift and peak power of the fs pulse seed. The paper is laid out as follows. In section 2, we start with the appropriate theoretical model for exploring fs seeded SC. In section 3, we delineate the detailed numerical results and discussion. Sub-section 3.1 addresses the role of a weak fs pulse seed on $\mathrm{CW}$ pumped SC generation. In sub-section 3.2, we analyze the influence of frequency shift over CW pumped SC generation. Besides, we also discuss the impact of peak power of the seed in subsection 3.3. Finally, we summarize the results in section 4 .

\section{Theoretical Model}

The generalized nonlinear Schrödinger equation (GNLSE) is used to model the SC generation in the optical fiber [32]:

$$
\begin{aligned}
& \frac{\partial A}{\partial \mathrm{z}}+i \frac{\beta_{2}}{2} \frac{\partial^{2} A}{\partial t^{2}}-\frac{\beta_{3}}{6} \frac{\partial^{3} A}{\partial t^{3}}+\frac{\alpha}{2} A= \\
& \left.\left.\left.i \gamma\left(1+i \tau_{\text {shock }} \frac{\partial}{\partial t}\right)(A z, t) \int_{-\infty}^{+\infty} R t^{\prime}\right) \mid A z, t^{\prime}-t^{\prime}\right)\left.\right|^{2} d t^{\prime}\right),
\end{aligned}
$$

where $A z, t)$ is the slowly varying amplitude of the pulse envelope, $t$ is the propagation time, $z$ is the propagation distance. Here, the parameters $\alpha, \beta_{2}, \beta_{3}$ and $\gamma$ are the loss, second-order dispersion, third-order dispersion and nonlinear coefficients of the fiber, respectively. $\tau_{\text {shock }}=\tau_{0}=1 / \omega_{0}$ where $\omega_{0}$ is the pump center frequency. The Raman response function is given by: $R(t)=\left(1-f_{R}\right) \delta(t)+f_{R} h_{R}(t)$ where $f_{R}=0.18$ is the fractional contribution of the delayed Raman response and $\delta(t)$ is the Dirac delta function. Here, ${ }^{h_{R}}$ is represented by the formula in [33] with $\tau_{1}, \tau_{2}$ being $12.2 \mathrm{fs}$ and $32 \mathrm{fs}$, respectively. The pump source is considered as an ideal $\mathrm{CW}$ of constant power $P_{0}=6 \mathrm{~W}$ in time domain and a center wavelength of $\lambda_{0}=1486 \mathrm{~nm}$. The input pulse shot noise is modeled semi-classically by adding a noise seed of one photon per mode with random phase on each spectral discretization bin [26] and the noise amplitude is assumed to be $10^{-6}$ of the pump amplitude. A 400 meters long highly nonlinear, dispersion shifted fiber is considered here, and its fiber parameters are assumed to be $\beta_{2}=-0.17 \mathrm{ps}^{2} / \mathrm{km}$, $\beta_{3}=0.0393 \mathrm{ps}^{3} / \mathrm{km}$ (measured in [34]), the nonlinear coefficient $\gamma=15 / \mathrm{W} / \mathrm{km}$, and $\alpha=10 \mathrm{~dB} / \mathrm{km}$. The femtosecond pulse seed is given by, $A_{\text {sed }}=\sqrt{\varepsilon P_{0}} \exp (-i \Omega t) \times \exp \left(-t^{2} / 2 / T_{0}^{2}\right)$. Here, $\varepsilon P_{0}$ is the peak power of the seed, $\varepsilon$ is the proportion of peak power of seed to peak power of the pump, $\Omega$ is the frequency shift of the pump and we assume a transform-limited Gaussian pulse as the seed with a pulse width $T_{0}$ of 200 fs. The position of the seed in wavelength is calculated by, $\lambda_{\text {seed }}=1 /\left(1 / \lambda_{0}+\Omega / 2 / \pi / c\right)$ where $\lambda_{0}$ is the center wavelength of the pump and $c$ is the speed of light in vacuum. Here, GNLSE is numerically solved by the splitstep method and 4th order Runge-Kutta method. Sampling points of 131072 and a time window of 512 ps are used. For the numerical parameters used, the resolution is $\sim 0.015 \mathrm{~nm}$, and this is also the linewidth of the CW pump. The resolution is also comparable to that of Yokogawa optical spectrum analyzer AQ 6370D $(0.02 \mathrm{~nm}$ from $1520 \mathrm{~nm}$ to $1620 \mathrm{~nm}$ ). For a better illustration, the CW pump is shown in both spectral and time domain in Fig. 4, and the CW pump together with the femtosecond pulse seed are displayed in Fig. 5 for both spectral and time domain.

\section{Numerical Results and Discussion}

We present the detailed numerical results on weak coherent fs pulse seed on the CW pumped SC generation in terms of bandwidth, coherence and SNR. We first provide a comprehensive insight on how a weak seed influences the properties of the generated SC. Then, we tune the frequency shift of the seed to investigate the effective range of the frequency shifts. Finally, we also address the nature of the CW pumped SC when the peak power of seed is varied. Thus, based on the results, we present an idea on the choice of frequency shift and the desired peak power of the seed for the effective generation of $\mathrm{CW}$ pumped SC.

\subsection{Effects of a weak femtosecond pulse seed}

In order to understand the importance of a seed pulse, first, we carry out the $\mathrm{CW}$ pumped $\mathrm{SC}$ without a seed pulse. Then, we introduce a weak fs seed, which, results in enhancing the spectral bandwidth and stability of CW pumped SC.

Figure 1(a) represents the $\mathrm{CW}$ pumped $\mathrm{SC}$ in the absence of a seed pulse. Here, 500 individual events were carried out with different random noises. In Fig. 1(a), the gray lines show every individual event and the average is represented 
by the black line. Here, quantitatively we set $-50 \mathrm{~dB}$ as a standard line. We find that the unseeded SC spectrum spans from 1363 to $1645 \mathrm{~nm}$ with a bandwidth of $282 \mathrm{~nm}$. As shown in Fig. 1(b), in contrast to the unseeded one, the SC spectrum spans from 1228 to $1872 \mathrm{~nm}$ with a bandwidth of $644 \mathrm{~nm}$ when a weak fs pulse seed is introduced. Thus, the seed pulse helps in enhancing the more spectra of $362 \mathrm{~nm}$ when compared to unseeded one. The spectral broadening stems from accumulative MI that leads to the breakup of CW into fundamental solitons. In the spectral broadening process, the linear dispersion and some nonlinear effects, namely, self-phase modulation and Raman scattering act together to produce new spectral components continuously. Therefore, the spectral broadening takes place continuously and finally results in SC generation. As MI is driven by noise, the unstable distribution of peak power probability emerges after the nonlinear process.

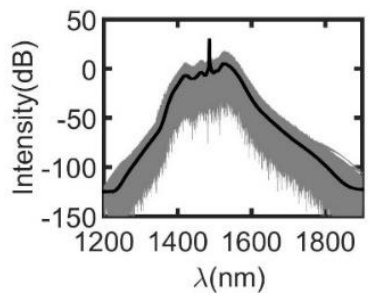

(a)

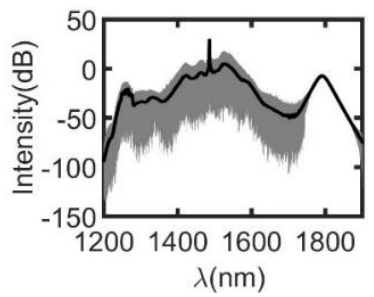

(b)
Fig. 1. Comparison between unseeded (a) and seeded SC (b) in SC spectra. For both unseeded and seeded SC, 500 individual simulations with different noise seeds are used.

Now, we turn to analyze the important characteristics of SC, namely, coherence and SNR. Coherence is used to characterize phase stability while SNR is used to characterize spectral intensity stability. Figure 2 shows the coherence (black dashed line) and SNR (blue solid line) for both unseeded SC in Fig. 2(a) and seeded SC in Fig. 2(b). In Fig. 2(a), the coherence of unseeded SC is almost zero for all the wavelengths except the center wavelength whose coherence is close to one. The poor coherence of unseeded SC originates from the phase fluctuations. As depicted in Fig. 2(b), the coherence of the seeded SC gets improved considerably, especially, at the shorter wavelength regime i.e., from 1200 to $1400 \mathrm{~nm}$ where dispersive waves exist. Blue solid lines in Figs. 2(a) and 2(b) represent the SNR for unseeded and seeded cases, respectively. When a weak seed is introduced, the SNR gets enhanced to a larger extent at longer wavelengths. The maximum value of SNR is 60 for a seeded one whereas it is extremely less for unseeded one. For instance, the average SNR for unseeded and seeded are 0.91 and 23.84, respectively, for a wavelength range from 1700 to $1900 \mathrm{~nm}$. From the above illustrations, one can understand that after applying a weak fs pulse seed, both phase and intensity stability of SC get improved. This can be understood by the fact that the seed enhances the formation of solitons in the longer wavelength regime and dispersive waves in the shorter wavelength regime.

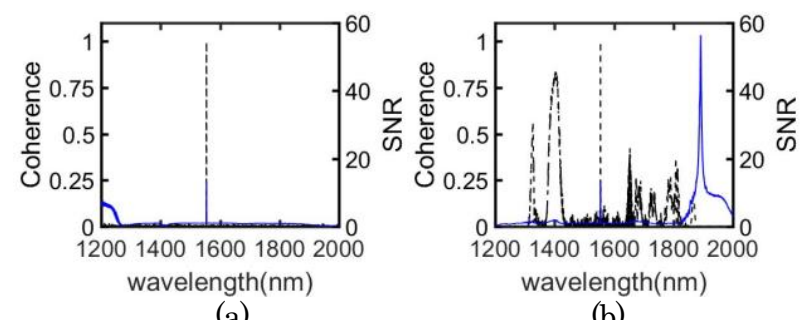

(a)

(b)

Fig. 2. Coherence (black dashed line) and SNR (blue solid line) for (a) unseeded and (b) seeded SC coherence.

In order to understand the influence of a seed on $\mathrm{CW}$ pumped SC, in addition to the above analysis, we now explore the evolution spectra of $\mathrm{CW}$ pumped SC. Figures 3(a) and 3(b) show the generation of unseeded $\mathrm{CW}$ pumped $\mathrm{SC}$ where MI is mainly driven by random noise. On the other hand, Figs. 3(c) and 3(d) depict the spectral evolution of seeded SC generation from 0 to $400 \mathrm{~m}$ at every $50 \mathrm{~m}$. In Fig. 3(a), the unseeded SC at $50 \mathrm{~m}$ has two side lobes on either side. For the seeded SC, this first order MI again produces second order assisted by FWM and goes on until on the right-side it started broadening due to Raman scattering. In case of seeding this MI order goes quite a good number, and the same is witnessed by several side lobes in the spectrum at $50 \mathrm{~m}$ in Fig. 3(d). In the seeded SC, instead of noise, the seed actively participates in the FWM process and hence reinforces the spectral broadening. Thus, seeded SC has a wider and a smoother spectrum. After the breakup of $\mathrm{CW}$ into fundamental solitons, the soliton collisions lead to the formation of Raman soliton at longer wavelength regime. Further, a part of solitons is blue-shifted to the shorter wavelength regime, which, results in the formation of dispersive waves. Here, the seed not only speeds up the MI process for converting $\mathrm{CW}$ into fundamental solitons, but also enhances the formation and stability of Raman solitons and dispersive waves. As the formation of solitons is due to the weak fs pulse seed, the phase and intensity fluctuations are suppressed. Consequently, after generating SC, both the phase and intensity stability get enhanced. Thus, a seed not only improves the bandwidth and filtered peak power distribution stability but also considerably enhances the coherence and SNR.

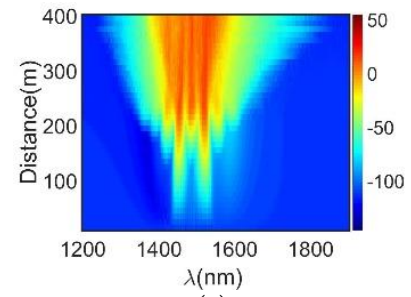

(a)

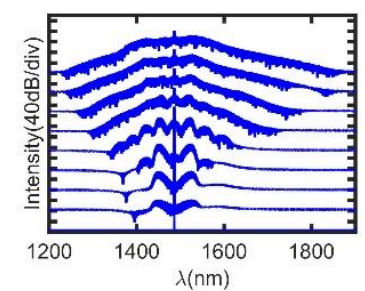

(b) 


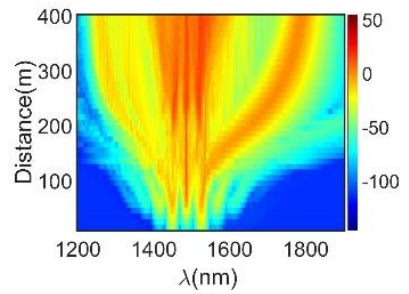

(c)

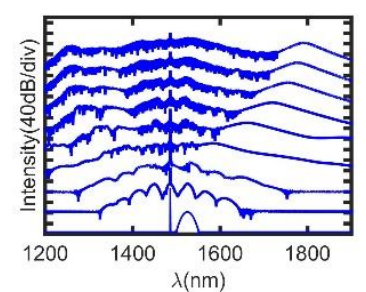

(d)
Fig. 3. Comparison between (a \& b) unseeded SC generation evolution of spectra; (c \& d) seeded SC generation evolution of spectra. The spectra in (b $\&$ d) are plotted from $z=0$ to $z=400 \mathrm{~m}$ at every $50 \mathrm{~m}$.

In order to look into the details of the SC evolution, we show the enlarged figures of Fig. 3 at fiber lengths of $0 \mathrm{~m}, 1 \mathrm{~m}, 5$ $\mathrm{m}$ and $10 \mathrm{~m}$ for both unseeded SC in Fig. 4 and seeded SC in Fig. 5. For the unseeded SC, initially at $z=0$, the pump source is a narrow linewidth $\mathrm{CW}$ at $1486 \mathrm{~nm}$ and in the time domain it is $6 \mathrm{~W}$ if the background noise is ignored. Until the fiber length of $10 \mathrm{~m}$, the spectrum almost maintains the input shape, and the corresponding behaviour in the time domain is also similar to the initial condition. For the seeded SC, initially at $\mathrm{z}=0$, the spectrum is shown as a narrow linewidth $\mathrm{CW}$ and a fs pulse which covers nearly $80 \mathrm{~nm}$ in spectrum. The initial phase of pump propagation in the fiber is marked by the development of MI. For instance, at the short fiber length of $1 \mathrm{~m}$, there are two definite peaks positioned symmetrically on both side of the pump wavelength. Development of MI in time domain leads to oscillations. As the pump radiation propagates through the fibre, oscillation amplitude grows and the energy travels in the form of successive pulses.
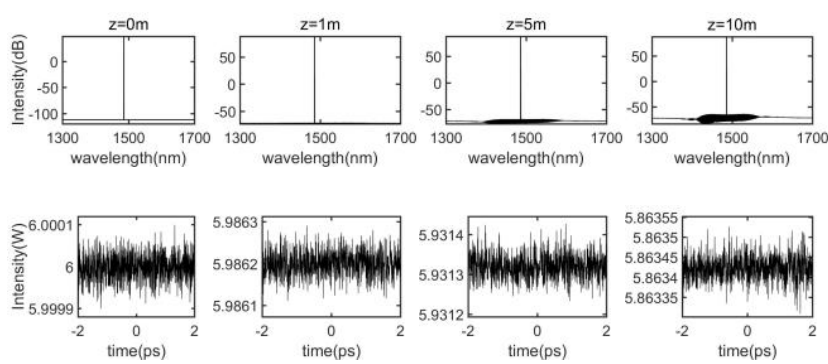

Fig 4. Dynamics of SC generated by a CW pump without seed: The variation of spectra (upper row) and intensity (lower row) against time for a propagation distance of $\mathrm{z}$. The physical parameters remain the same as in Fig. 3.
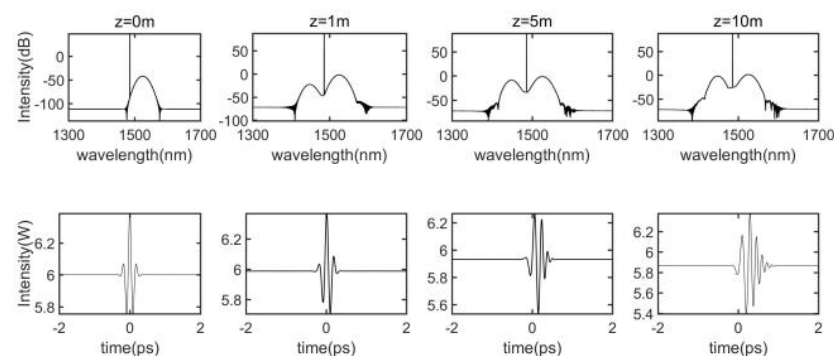

Fig 5. Dynamics of SC generated by a CW pump with a weak fs pulse seed: The variation of spectra (upper row) and intensity (lower row) against time for a propagation distance of $\mathrm{z}$. The physical parameters remain the same as in Fig. 3.

\subsection{The influence of frequency shift}

Having realized the importance of the seed pulse in $\mathrm{CW}$ pumped SC, we now plan to investigate the influence of the frequency shift of the seed on $\mathrm{CW}$ pumped SC generation. By varying the relative position of the seed, we intend to optimize the desired frequency shift for the effective SC generation.

We show the spectral evolution of single simulations for selected frequency shift when set $1486 \mathrm{~nm}$ pump center wavelength, $P_{0}=6 \mathrm{~W}$ and a $P_{S}=0.001 \mathrm{P}_{0}$, and other parameters keep the same as before. In Figs. 6(a)-6(e), 200 simulations with different random noise are carried out. To start with, first, we assume that the seed is exactly located at the center wavelength, i.e., the frequency shift, $\Omega$, is $0 \mathrm{THz}$. The corresponding results are shown in Fig. 6(a), which, are almost the same as unseeded one. In this case, MI is still mainly driven by noise because the peak of pump and seed coincide together in spectrum. Thus, in the beginning of spectral broadening, the seed almost has no effect on MI and its role is extremely limited in the whole nonlinear process. Secondly, if the seed is located in between the center wavelength and MI gain peak, then the seed plays a vital role in FWM which can be seen from the evolution of the spectral broadening as illustrated in Fig. 6(b). Especially, at the longer wavelength regime, the Raman solitons are particularly enhanced and the value of SNR exceeds 50 . However, the coherence remains as poor as unseeded one. Though the seed starts influencing the SC generation, its role is restricted in this case, as the seed does not dominantly initiate the MI. When the frequency shift is closer to MI gain peak, we observe the two peaks in the spectrum at longer wavelengths that can be explained by irregular energy transfer. Correspondingly, the two peaks are observed in SNR at longer wavelengths and the coherence also gets improved to some extent. In the third case, the frequency shift exactly matches with that of the frequency of MI gain peak, which is calculated by $\Omega_{\mathrm{M}}= \pm \sqrt{2 \gamma P_{0} /\left|\beta_{2}\right|}$. In this case, the seed dominantly initiates the FWM and the spectral broadening is highly enhanced. Further, the coherence gets considerable improvement, especially, at the shorter wavelengths where dispersive waves exist. Thus, as shown in Fig. 6(c), the bandwidth, coherence and SNR get enhanced a lot. In the fourth case, the seed is moved a little far away from center wavelength and MI gain peak with a frequency shift of $-45 \mathrm{THz}$. In this case, we find that all the statistical characteristics decrease as portrayed in Fig. 6(d) which can be understood by the restricted gain broadening and this is similar to the second case [refer Fig. 6(b)]. Finally, the seed is moved further resulting in a frequency shift of $-50 \mathrm{THz}$. Under this condition, the seed loses the control to generate SC gradually as shown in Fig. 6(e). Now, the properties of $\mathrm{SC}$ resemble the same as unseeded event. In this region, the 
MI gain is close to 0 and hence the seed would not participate in the MI process. Eventually, the seed ceases to hold control. The aforementioned discussions boil down to four important regimes for the frequency shift of the seed and they are:

(i) Condition I: $0<\Omega<0.5 \Omega \mathrm{M}$, the seed is relatively far away from MI gain peak, and the detailed results are shown in Fig. 6(b). Here, noise plays an important role in the MI process. The seed starts influencing but its role is limited. The spectrum broadens wider and SNR gets enhanced to a larger extent. However, the coherence becomes very poor and it remains as the unseeded events as shown in Fig. 6(a).

(ii) Condition II: $0.5 \Omega_{\mathrm{M}}<\Omega<\Omega_{\mathrm{M}}$, the seed is close to MI gain peak. Owing to rigorous FWM process, there is irregular energy transfer between soliton collisions. Consequently, two peaks are observed at longer wavelengths. In this case, in addition to wider spectrum, the other characteristics, namely, coherence and SNR get improved to a larger extent as illustrated in Fig. 6(c).

(iii) Condition III: $\Omega_{\mathrm{M}}<\Omega<1.5 \Omega_{\mathrm{M}}$, the seed exactly lies at the peak of the MI peak gain. In this case, the seed dominantly initiates the FWM and the spectral broadening is highly enhanced. Hence, as shown in Fig. 6(c), the bandwidth, coherence and SNR get enhanced to a larger extent. Thus, this is treated as a desired condition for the $\mathrm{CW}$ pumped SC generation.

(iv) Condition IV: $1.5 \Omega_{\mathrm{M}}<\Omega$, the seed is far away from center wavelength and MI gain peak. Hence, as shown in Fig. 6(e) the seed does not actively participate in MI and it ceases to hold control on SC generation.

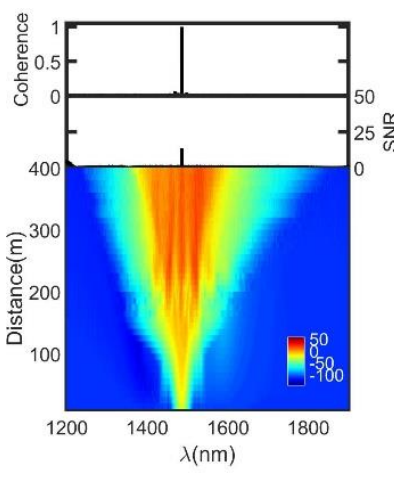

(a)

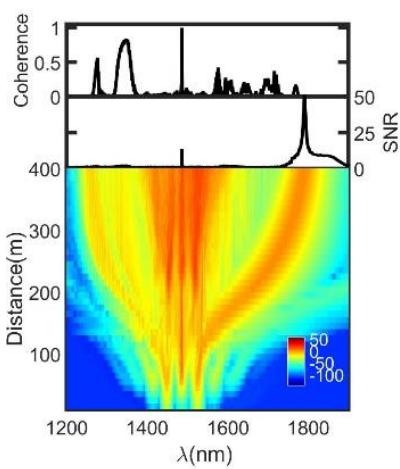

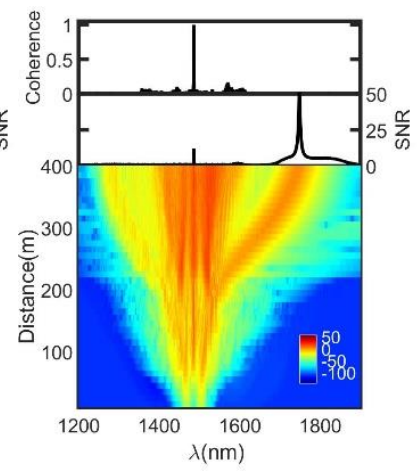

(b)

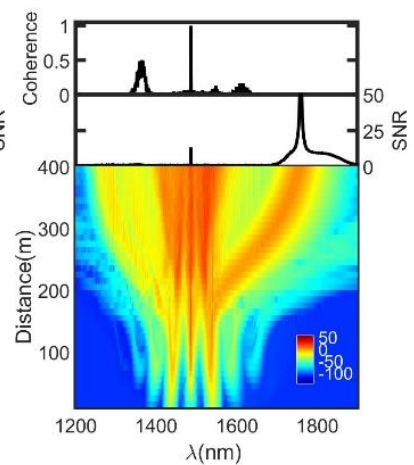

(c)

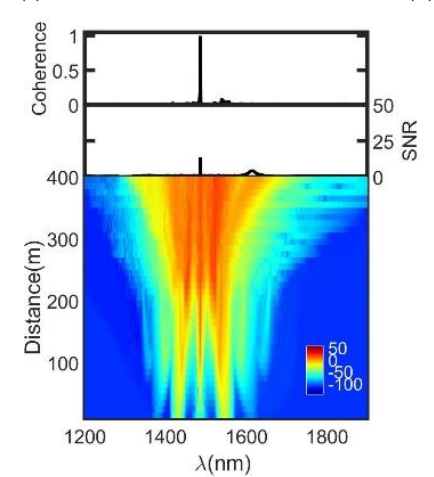

(e)

Fig. 6. Single-shot simulations of $0.1 \%$ seed at frequency shifts (a-e) $0,-15$, $-32.5,-45$ and $-50 \mathrm{THz}$, respectively. The top rows in (a-e) show the ensemble calculated spectral, coherence and SNR. (a \& b, b \& c, c \& d and d $\&$ e) are in the range of i, ii, iii and iv respectively.

\subsection{The influence of seed peak power}

In this sub-section, we investigate the influence of peak power of the seed on CW pumped SC. Undoubtedly we find that the peak power of the seed does significantly influence the nonlinear process from the beginning where CW breaks into solitons. We set the peak power of the seed to be $10 \%$, $1 \%, 0.1 \%$ and $0.01 \%$ of $\mathrm{P}_{0}$, and the frequency shift is fixed at $-32.5 \mathrm{THz}$ that corresponds to peak of MI gain peak. The important characteristics such as spectra, coherence, SNR and distribution of peak power will change with the change of the peak power of the seed. For instance, we set $\varepsilon$ to be 0.1 and remove the $\mathrm{CW}$. Then, the fs pulse generates almost 200 nm continuum. Thus, we consider $\varepsilon=0.1$ is the ultimate value of the femtosecond seed.

Figures 7 (a-d) show the seeded SC when the peak power of the seed is varied. For all the chosen peak powers of the seed, the spectrum broadens wider than the unseeded events. After the first stage of $\mathrm{CW}$ pumped SC generation, the soliton collision starts and it results in energy transfer between the solitons. When the peak power of the seed is relatively high as shown in Figs. 7(a) and 7(b), the energy transfer takes place rigorously. As a consequence, the spectrum broadens widely and there are two peaks in the long-wavelength regime. When the peak power of the seed is decreased further, the energy transfer process becomes slow and hence the bandwidth is relatively narrow as shown in Figs. 7(c) and 7(d). From Figs. 7(a-d), for the peak power of $10 \%, 1 \%, 0.1 \%$ and $0.01 \%$ of $P_{0}$, the corresponding bandwidths are $669 \mathrm{~nm}, 649 \mathrm{~nm}, 628 \mathrm{~nm}$, and $614 \mathrm{~nm}$, respectively. Thus, with the decrease of the peak power of the seed, the spectral bandwidth decreases. 


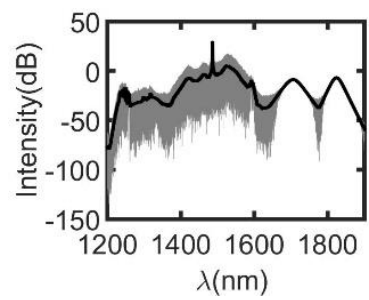

(a)

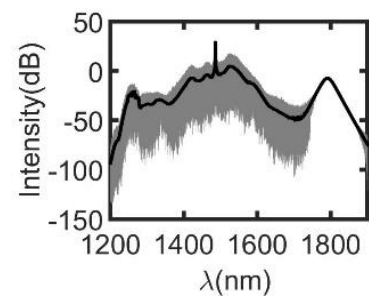

(c)

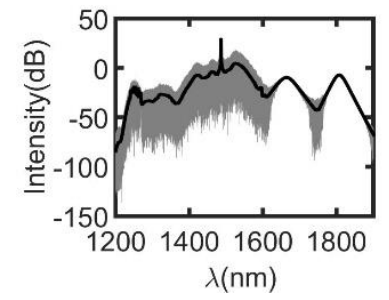

(b)

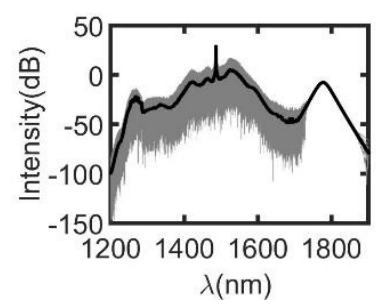

(d)
Fig. 7. Spectrum of SC when (a) $\varepsilon=10 \%$; (b) $\varepsilon=1 \%$; (c) $\varepsilon=0.1 \%$; (d) $\varepsilon=0.01 \%$

In addition to the SC spectrum analysis, we also provide the statistic consequences of SC to investigate the stability. Figures 8 (a-d) represent the coherence (black dashed line) and SNR (blue solid line) of SC for different seed power. When the seed power is relatively high, i.e., when $\varepsilon_{\text {are }} 10 \%$ and $1 \%$, the coherence gets enhanced for a wide range of wavelengths as shown in Figs. 8(a) and 8(b). Two peaks are observed at both longer and shorter wavelengths. This can be understood by the fact that when the seed power is relatively high, the control will be enhanced. In other words, the seed plays an indispensable role than noise. In contrast to the above, if the seed power is relatively low, then the control of the seed is restricted as depicted in Figs. 8(c) and 8(d). Therefore, the coherence decreases with the decrease of the peak power of the seed. In Figs. 8(c) and 8(d), though the seed power is 10 times weaker, the spectrum looks almost the same and bandwidths are $628 \mathrm{~nm}$ and $614 \mathrm{~nm}$, respectively. In the case of coherence, when $\varepsilon_{\text {is }}$ ten times weaker, the coherence decreases drastically. For the seed power of $10 \%, 1 \%, 0.1 \%$ and $0.01 \%$, the corresponding coherences [3] are $0.1,0.088,0.085$ and 0.083 , respectively. Thus, with the decrease of the seed power, the overall coherence decreases. The next step is to determine the corresponding SNR for various values of the peak power of the seed. When the peak power of the seed is relatively high, there are two peaks at longer wavelengths as shown in Figs. 8(a) and 8(b). The peak value of the SNR diminishes when the peak power of seed is decreased. Finally, only one peak is observed at longer wavelengths. As the influence of SNR concentrates in the longer wavelength regime, we report the overall SNR for a longer wavelength range from 1600 - 1900 $\mathrm{nm}$ and they are 21.3, 17.1, 22.7 and 21.5. For the unseeded events, the peak value of the SNR is located at the center wavelength. Peak value of the SNR is 13 and the overall SNR at longer wavelengths is 0.8. Thus, the SNR can relatively be enhanced at longer wavelengths.

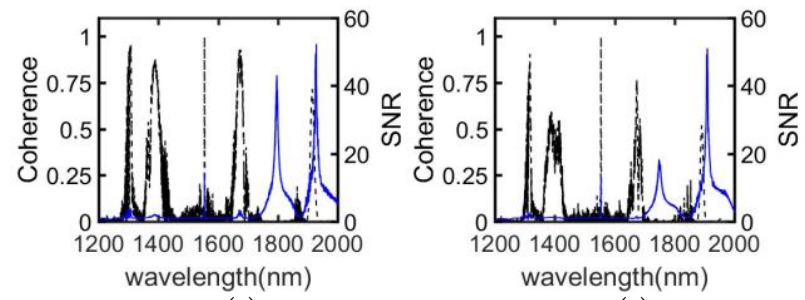

(a)

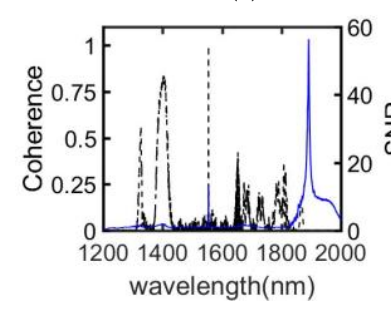

(c) (b)

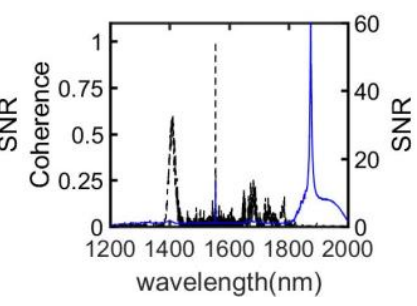

(d)
Fig. 8. Coherence (black dashed line) and SNR (blue solid line) of SC when (a) $\varepsilon=10 \%$; (b) $\varepsilon=1 \%$; (c) $\varepsilon=0.1 \%$; (d) $\varepsilon=0.01 \%$

\section{Conclusions}

In this work, we have numerically investigated the role of a femtosecond seed pulse in a continuous wave pumped supercontinuum generation. Initially, the CW pumped SC is formed by MI process which has been initiated by random noise. We have observed the existence of instabilities in $\mathrm{CW}$ pumped SC generation. Hence, the resulting SC by this MI process possesses the poor characteristics of phase, intensity and energy distribution stability. In order to improve the aforementioned characteristics, we have launched an extremely weak coherent femtosecond pulse seed to assist the CW pumped SC generation. Thus, the MI is initiated by this weak femtosecond seed pulse rather than the random noise. We found that the seed assisted process helps in generating the wider SC with the augmented characteristics of a high coherence and a large SNR.

Further, in order to gain more insight, we have also analyzed the properties of $\mathrm{CW}$ pumped SC by varying the characteristics of the seed pulse, namely, the frequency shift and peak power. With respect to the location of the seed pulse, we have identified four conditions for the frequency shift. Simulations results detail the influence of the frequency shifts and the peak power of the fs pulse seed in the SC generation. Based on the coherence and SNR properties, we have optimized the following frequency range, $\Omega_{\mathrm{M}}<\Omega<$ $1.5 \Omega \mathrm{M}$, as the effective range of frequency shift for the seed pulse. Besides, we have explored the evolution of the SC spectra by varying the peak power. It has been found that the bandwidth decreases when the peak power is decreased.

In a nutshell, by introducing an extremely weak coherent femtosecond pulse seed to assist the CW pumped SC generation, we could effectively control the spectral instabilities. Based on the detailed numerical results, we claim that we have been able to generate the wider SC with the enriched qualities. Thus, we anticipate that the reported 
SC source would highly be useful in several branches of science, engineering and medicine as an effective source.

\section{Acknowledgements}

This work was supported by the National Science Foundation of China (No. Project 61675008). Nakkeeran wishes to thank Santander Mobility Award for his visit to Peking University Shenzhen Graduate School.

\section{References}

[1] Marks D L, Oldenburg A L, Reynolds J J and Boppart S A 2002 Study of an ultrahigh-numerical-aperture fiber continuum generation source for optical coherence tomography Opt. Lett. 27 2010-2012

[2] Washburn B R and Newbury N R 2004 Phase, timing, and amplitude noise on supercontinua generated in microstructure fiber Opt. Express 12 2166-2175

[3] Dudley J M, Genty G and Coen S 2006 Supercontinuum generation is photonic crystal fiber Rev. Mod. Phys 78 11351184

[4] Alfano R R The Supercontiuum Laser Source: Fundamentals with Updated References, 2 nd ed. (Springer-Verlag, New York, 2006).

[5] Genty G, Lehtonen M, Ludvigsen H and Kaivola M 2004 Enhanced bandwidth of supercontinuum generated in microstructured fibers Opt. Express 12 3471-3480

[6] Dudley J M, Provino L, Grosard N, Maillotte H, Windeler R S, Eggleton B J and Coen S 2002 Supercontinuum generation in air-silica microstructured fibers with nanosecond and femtosecond pulse pumping J. Opt. Soc. Am. B 19 765-771

[7] Coen S, Chau A H L, Leonhardt R, Harvey J M, Knight J C, Wadsworth W J and Russell P St J 2002 Supercontinuum generation by stimulated Raman scattering and parametric fourwave mixing in photonic crystal fibers J. Opt. Soc. Am. B 19 753-764

[8] Avdokhin A V, Popov S V and Taylor J R 2003 Continuouswave, high-power, Raman continuum generation in holey fibers Opt. Lett. 28 1353-1355

[9] Nicholson J W, Abeeluck A K, Headley C, Yan M F, and Jørgensen C G 2003 Pulsed and continuous-wave supercontinuum generation in highly nonlinear, dispersionshifted fibers Appl. Phys. B 77 211-218

[10]González-Herráez M, Martin-López S, Corredera P, Hernanz M L and Horche P R 2003 Supercontinuum generation using a continuous-wave Raman fiber laser Opt. Commun. 226323 328

[11]Abeeluck A K and Headley C 2004 Supercontinuum growth in a highly nonlinear fiber with a low-coherence semiconductor laser diode Appl. Phys. Lett. 85 4863-4865

[12]Abeeluck A K and Headley C 2005 Continuous-wave pumping in the anomalous- and normal-dispersion regimes of nonlinear fibers for supercontinuum generation Opt. Lett. 30 61-63

[13]Ranka J K, Windeler R S and Stentz A J 2000 Visible continuum generation in air-silica microstructure optical fibers with anomalous dispersion at $800 \mathrm{~nm}$ Opt. Lett. 25 25-27
[14]Corwin K L, Newbury N R, Dudley J M, Coen S, Diddams S A, Weber K and Windeler R S 2003 Fundamental noise limitations to supercontinuum generation in microstructure fiber Phys. Rev. Lett., 90, 113904

[15]Matos C J S de, Popov S V and Taylor J R 2004 Temporal and noise characteristics of continuous-wave-pumped continuum generation in holey fibers around 1300nm Appl. Phys. Lett. 85 2706-2708

[16]Hsiung P L, Chen Y, Ko T, Fujimoto J G, Matos C J S de, Popov S V, Taylor J R and Gapontsev V P 2004 Optical coherence tomography using a continuous-wave, high-power, Raman continuum light source Opt. Express 12 5287-5295

[17]Prabhu M, Taniguhci A, Hirose S, Lu J, Musha M, Shirakawa A and Ueda K 2003 Supercontinuum generation using Raman fiber laser Appl. Phys. B. 77 205-210

[18]Champert P A, Couderc V and Barthelemy A 2004 1.5-2.0- $\mu \mathrm{m}$ multiwatt continuum generation in dispersion-shifted fiber by use of high-power continuous-wave fiber source IEEE Photon. Technol. Lett. 16 2445-2447

[19]Abeeluck A K, Headley C, and Jorgensen C G 2004 Highpower supercontinuum generation in highly nonlinear, dispersion-shifted fibers by use of a continuous-wave Raman fiber laser Opt. Lett. 29 2163-2165

[20]Sylvestre T, Vedadi A, Maillotte H, Vanholsbeeck F and Coen S 2006 Supercontinuum generation using continuous-wave multiwavelength pumping and dispersion management Opt. Lett. 31 2036-2038

[21]Abrardi L, Martin-Lopez S, Carrasco-Sanz A, Corredera P, Hernanz M L and Gonzalez-Herraez M 2007 Optimized AllFiber Supercontinuum Source at $1.3 \mu \mathrm{m}$ Generated in a Stepwise Dispersion-Decreasing-Fiber Arrangement IEEE Photon. Technol. Lett. 25 2098-2012

[22]Mussot A, Lantz E, Maillotte H, Sylvestre T, Finot C and Pitois S 2004 Spectral broadening of a partially coherent CW laser beam in single-mode optical fibers Opt. Express 12 2838-2843

[23]Vanholsbeeck F, Martin-Lopez S, Gonzalez-Herraez M and Coen S 2005 The role of pump incoherence in continuous-wave supercontinuum generation Opt. Express 13 6615-6625

[24]Kobtesv S M and Smirnov V 2005 Modelling of high-power supercontinuum generation in highly nonlinear, dispersion shifted fibers at CW pump Opt. Express 13 6912-6918

[25]Frosz M H, Bang O and Bjarklev A 2006 Soliton collision and Raman gain regimes in continuous-wave pumped supercontinuum generation Opt. Express 14 9391-9407

[26]Mussot A, Kudlinski A, Kolobov M, Louvergneaux E, Douay M and Taki M 2009 Observation of extreme temporal events in CW-pumped supercontinuum Opt. Express 17 17010-17015

[27]Lu F and Knox W 2004 Generation of a broadband continuum with high spectral coherence in tapered single-mode optical fibers Opt. Express 12 347-353

[28]Dudley J M and Coen S 2002 Coherence properties of supercontinuum spectra generated in photonic crystal and tapered optical fibers Opt. Lett. 27 1180-1182

[29]Kues M, Brauckmann N, Walbaum T, Groß P and Fallnich C 2009 Nonlinear dynamics of femtosecond supercontinuum generation with feedback Opt. Express 17 15827-15841

[30]Solli D R, Ropers C and Jalali B 2008 Active Control of Rogue Waves for Stimulated Supercontinuum Generation Phys. Rev. Lett. 101 275-278 
[31] Sorensen S T, Bang O, Wetzel B and Dudely J M 2012 Describing supercontinuum noise and rogue wave statistics using higher-order moments Opt. Commun 285 2451-2455

[32] Agrawal G P, Nonlinear Fiber Optics (4th Edition), (Academic, 2007).

[33]Blow K J and Wood D 1989 Theoretical Description of Transient Stimulated Raman Scattering in Optical Fibres IEEE J. Quantum Electron. 25 2665-2673

[34]Abeeluck A K, Headley C, Jorgensen C G 2004 High-power supercontinuum generation in highly nonlinear dispersionshifted fibers by use of a continuous-wave Raman fiber laser Opt. Lett. 29 2163-2165 